\title{
Chest CT-derived pulmonary artery enlargement at the admission predicts overall survival in COVID-19 patients: insight from 1461 consecutive patients in Italy
}

\author{
Antonio Esposito ${ }^{1,2}$ (D) Anna Palmisano ${ }^{1,2} \cdot$ Marco Toselli $^{3} \cdot$ Davide Vignale $^{1,2} \cdot$ Alberto Cereda $^{3}$. \\ Paola Maria Vittoria Rancoita ${ }^{2,4}$ - Riccardo Leone ${ }^{1,2}$ - Valeria Nicoletti ${ }^{1,2}$. Chiara Gnasso ${ }^{1,2}$. Alberto Monello ${ }^{5}$. \\ Andrea Biagi ${ }^{5}$. Piergiorgio Turchio ${ }^{5}$. Giovanni Landoni ${ }^{2,6}$. Guglielmo Gallone ${ }^{7}$. Giacomo Monti ${ }^{6}$. Gianni Casella ${ }^{8}$. \\ Gianmarco lannopollo ${ }^{8}$. Tommaso Nannini ${ }^{8}$. Gianluigi Patelli ${ }^{9}$. Luisa Di Mare ${ }^{9}$. Marco Loffi $^{10}$. Pietro Sergio ${ }^{10}$. \\ Davide Ippolito ${ }^{11}$. Sandro Sironi ${ }^{12} \cdot$ Gianluca Pontone $^{13} \cdot$ Daniele Andreini $^{13}$. Elisabetta Maria Mancini ${ }^{13}$. \\ Clelia Di Serio ${ }^{2,4}$ • Francesco De Cobelli $^{1,2}$ • Fabio Ciceri ${ }^{2,14}$ • Alberto Zangrillo ${ }^{2,6}$ • Antonio Colombo ${ }^{3}$. \\ Carlo Tacchetti ${ }^{1,2} \cdot$ Francesco Giannini $^{3}$
}

Received: 8 September 2020 / Revised: 6 November 2020 / Accepted: 10 December 2020 / Published online: 23 December 2020

(C) The Author(s) 2020

\begin{abstract}
Objectives Enlarged main pulmonary artery diameter (MPAD) resulted to be associated with pulmonary hypertension and mortality in a non-COVID-19 setting. The aim was to investigate and validate the association between MPAD enlargement and overall survival in COVID-19 patients.

Methods This is a cohort study on 1469 consecutive COVID-19 patients submitted to chest CT within $72 \mathrm{~h}$ from admission in seven tertiary level hospitals in Northern Italy, between March 1 and April 20, 2020. Derivation cohort $(n=761)$ included patients from the first three participating hospitals; validation cohort $(n=633)$ included patients from the remaining hospitals. CT images were centrally analyzed in a core-lab blinded to clinical data. The prognostic value of MPAD on overall survival was evaluated at adjusted and multivariable Cox's regression analysis on the derivation cohort. The final multivariable model was tested on the validation cohort.

Results In the derivation cohort, the median age was 69 (IQR, 58-77) years and 537 (70.6\%) were males. In the validation cohort, the median age was 69 (IQR, 59-77) years with $421(66.5 \%)$ males. Enlarged MPAD ( $\geq 31 \mathrm{~mm})$ was a predictor of mortality at adjusted (hazard ratio, HR [95\%CI]: 1.741 [1.253-2.418], $p<0.001$ ) and multivariable regression analysis (HR [95\%CI]: 1.592 [1.154-2.196], $p=0.005)$, together with male gender, old age, high creatinine, low well-aerated lung volume, and high pneumonia extension $(\mathrm{c}-$ index $[95 \% \mathrm{CI}]=0.826[0.796-0.851])$. Model discrimination was confirmed on the validation cohort $(\mathrm{c}-$
\end{abstract}

Antonio Esposito and Anna Palmisano contributed equally to this work.

Antonio Esposito

esposito.antonio@hsr.it

1 Experimental Imaging Center, IRCCS San Raffaele Scientific Institute, Via Olgettina 60, Milan, Italy

2 School of Medicine, Vita-Salute San Raffaele University, Via Olgettina 58, Milan, Italy

3 GVM Care \& Research Maria Cecilia Hospital, Cotignola, Italy

4 Centro Universitario di Statistica per le Scienze Biomediche, Vita-Salute San Raffaele University, Milan, Italy

5 Guglielmo da Saliceto Hospital, Piacenza, Italy

6 Anesthesia and Intensive Care Department, IRCCS San Raffaele Scientific Institute, Milan, Italy
Division of Cardiology, Department of Internal Medicine, Città della Salute e della Scienza, Turin, Italy

8 Ospedale Maggiore, Bologna, Italy

9 ASST Bolognini Hospital, Bergamo Est, Italy

10 Ospedale di Cremona, Cremona, Italy

11 San Gerardo Hospital, Monza, Italy

12 ASST Papa Giovanni XXIII, Bergamo, Italy

13 Centro Cardiologico Monzino IRCCS, Milan, Italy

14 Department of Hematology and Bone Marrow Transplantation, IRCCS San Raffaele Scientific Institute, Milan, Italy 
index $[95 \% \mathrm{CI}]=0.789[0.758-0.823])$, also using $\mathrm{CT}$ measurements from a second reader $(\mathrm{c}-\mathrm{index}[95 \% \mathrm{CI}]=0.790$ $[0.753 ; 0.825])$.

Conclusion Enlarged MPAD ( $\geq 31 \mathrm{~mm})$ at admitting chest CT is an independent predictor of mortality in COVID-19.

Key Points

- Enlargement of main pulmonary artery diameter at chest CT performed within $72 \mathrm{~h}$ from the admission was associated with a higher rate of in-hospital mortality in COVID-19 patients.

- Enlargement of main pulmonary artery diameter $(\geq 31 \mathrm{~mm})$ was an independent predictor of death in COVID-19 patients at adjusted and multivariable regression analysis.

- The combined evaluation of clinical findings, lung CT features, and main pulmonary artery diameter may be useful for risk stratification in COVID-19 patients.

Keywords COVID-19 $\cdot$ Thorax $\cdot$ Tomography, X-ray computed $\cdot$ Hypertension, pulmonary $\cdot$ Pulmonary artery

$\begin{array}{ll}\text { Abbreviations } & \\ \text { ARDS } & \text { Acute respiratory distress syndrome } \\ \text { CRF } & \text { Case report form } \\ \text { CRP } & \text { C-reactive protein } \\ \text { GGOs } & \text { Ground-glass opacities } \\ \text { LDH } & \text { Lactate dehydrogenase } \\ \text { LPAD } & \text { Left pulmonary artery diameter } \\ \text { MPAD } & \text { Main pulmonary artery diameter } \\ \text { PH } & \text { Pulmonary hypertension } \\ \text { RPAD } & \text { Right pulmonary artery diameter } \\ \text { RT-PCR } & \begin{array}{l}\text { Reverse transcriptase polymerase } \\ \text { chain reaction }\end{array} \\ \text { SARS-CoV-2 } & \text { Severe acute respiratory syndrome }\end{array}$

SARS-CoV-2 Severe acute respiratory syndrome coronavirus 2

\section{Introduction}

The coronavirus disease 2019 (COVID-19) rapidly invaded the world affecting millions of people and becoming a global health emergency.

Sparse autopsies on patients with COVID-19 have found interstitial pneumonia with diffuse alveolar damage, pulmonary arterioles thrombosis [1], and right ventricle (RV) dilation [2], suggesting that increased RV afterload due to endothelial injury [3] with lung vessel micro-thrombosis [4] might be a pathological driver in critical COVID-19 illness.

In two recent studies [5, 6] on 120 and 115 COVID-19 patients undergoing echocardiography, non-survivors displayed larger right-heart chambers, reduced RV function, and elevated pulmonary artery systolic pressure compared to survivors. In light of the possible impact of RV afterload on patients' outcome and treatment [7-9], more robust data coming from larger populations are needed to strengthen the current evidence. Data from echocardiography are scarce, because of technical [5] and practical limitations, mainly related to limited resources in a scenario of health emergency and to the need of reducing healthcare workers' exposure.
Chest CT has been widely adopted for COVID-19 pneumonia diagnosis [10], monitoring, and prognostication [11]. Beyond the extraction of parameters describing the features of lung involvement and its severity, the main pulmonary artery diameter (MPAD) can be easily measured from a chest CT scan. Enlarged MPAD on CT has been studied as a method for the screening and diagnosis of pulmonary hypertension $(\mathrm{PH})$ [12-15]. Reference values for healthy patients have been previously established [16] and showed excellent sensitivity for excluding PH [13]. In the pre-COVID-19 era, enlargement of MPAD resulted to be associated with PH severity at RV catheterization and to higher rate of mortality [13]. Hence, we hypothesized that the measurement of MPAD, a known marker of pulmonary hypertension, could be useful for risk stratification of COVID-19 patients.

The aim of the present study was to evaluate the prognostic value of MPAD enlargement at the admission chest $\mathrm{CT}$ on overall survival in COVID-19 patients considering a multivariable setting, and to validate the final multivariable model.

\section{Materials and methods}

\section{Study setting and participants}

This is a multicenter retrospective cohort study. Study participants were recruited among all adult patients (age 18 years or older) with confirmed RT-PCR for SARS-CoV-2, submitted to chest $\mathrm{CT}$ within $72 \mathrm{~h}$ from admission in seven tertiary level hospitals located in Northern Italy ((1) IRCCS San Raffaele Scientific Institute, Milan (OSR); (2) Guglielmo da Saliceto Hospital, Piacenza (PCZ); (3) Ospedale Maggiore, Bologna (BOL); (4) ASST Bolognini Hospital Bergamo Est (BGE); (5) Ospedale di Cremona (CRE); (6) San Gerardo Hospital, Monza (SGM); (7) Centro Cardiologico Monzino IRCCS, Milano (CCM)), between March 1 and April 20, 2020. Missing data on comorbidities or follow-up were considered exclusion criteria. The study was approved by the local ethics committees and written informed consent was obtained. 
Clinical data were collected by each center according to a centralized electronic case report form (CRF). Clinical data at the admission consisted of the following: demographic characteristics (sex and age), comorbidities (hypertension, diabetes, chronic lung disease, cardiovascular disease), and laboratory tests: white blood cell count (WBC), creatinine, Creactive protein $(\mathrm{CRP})$, lactate dehydrogenase ( $\mathrm{LDH})$, troponin I, interleukin-6, and D-dimer. Outcome data were orotracheal intubation and death.

All chest CT images were collected and analyzed in a single core-lab (Experimental Imaging Center, IRCCS San Raffaele Scientific Institute, Milan, Italy) blinded to clinical data.

The derivation cohort consisted of patients from the three hospitals (OSR, PCZ, BOL) that completed the CRF and shared chest CT images for centralized analysis within May 20, 2020, established as the first deadline for data collection. Patients from the remaining four participating hospitals (BGE, CRE, SGM, CCM), who provided data and CT images within the end of June, were used for the external validation. Prior to the analysis, data were cross-checked with medical charts and verified by data managers and clinicians for accuracy.

A total of 1469 consecutive patients fulfilled the inclusion criteria: 68 of the 829 patients of the derivation cohort and 7 of the 640 patients of the validation cohort were excluded for lacking data on comorbidities or follow-up (Fig. 1). The median follow-up was 51 days for the derivation cohort and 63 days for the validation cohort.

Complete demographic and clinical features of both cohorts are presented in Table 1.

\section{Outcomes}

The primary outcome was overall survival (OS) measured from admission to the emergency department. Secondary outcomes were in-hospital mortality and time-to-orotracheal intubation from admission to the emergency department.

\section{Chest CT scan}

All chest CT examinations were performed on multidetector scanners with at least 16 detector rows. The list of scanners and acquisition protocols are reported in Supplementary Materials (ESM 1). Briefly, all volumetric chest scans were sent to the core-lab where they were reformatted at a $2.5-\mathrm{mm}$ slice thickness without gap or overlap. Images were reconstructed with sharp kernel for lung parenchyma evaluation and a soft kernel for mediastinum evaluation, and they were visualized using a standard window (lung: width $1400 \mathrm{HU}$, center $-450 \mathrm{HU}$; mediastinum: width $350 \mathrm{HU}$, center 40 HU).

\section{Chest CT image analysis}

The analysis was performed by a radiologist with 9 years of experience in cardiothoracic imaging, blinded to all clinical data in order to reduce the possible bias in the analysis and to increase homogeneity and reliability of data. Parameters of lung involvement (residual respiratory lung reserve, pneumonia extension, and features) and pulmonary artery metrics were extracted (Fig. 2).

Residual respiratory lung reserve was measured as wellaerated lung volume, quantified using automatic software

Fig. 1 Enrollment flowchart

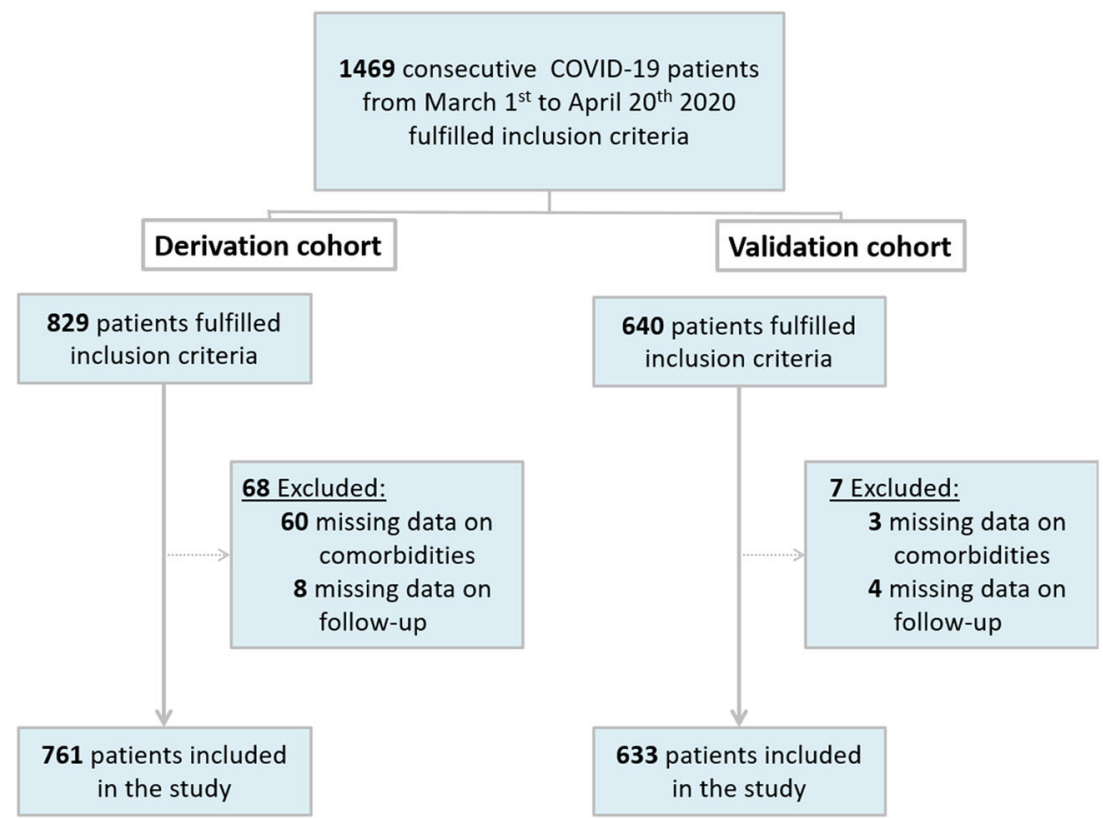


Table 1 Patient characteristics

\begin{tabular}{|c|c|c|c|c|}
\hline \multirow[b]{2}{*}{ Variables at the admission } & \multicolumn{2}{|c|}{ Derivation cohort } & \multicolumn{2}{|c|}{ Validation cohort } \\
\hline & $N$ & Median [IQR] & $N$ & Median [IQR] \\
\hline Age, years & 761 & $69.25[58.01-76.87]$ & 633 & $69.14[59.03-77.43]$ \\
\hline Male & 761 & $537(70.6 \%)$ & 633 & $421(66.5 \%)$ \\
\hline Hypertension, no. (\%) & 761 & $484(63.6 \%)$ & 633 & $285(45 \%)$ \\
\hline Diabetes, no. (\%) & 761 & $150(19.7 \%)$ & 633 & $119(18.8 \%)$ \\
\hline Known cardiovascular disease, no. $(\%)$ & 761 & $95(12.5 \%)$ & 633 & $129(20.4 \%)$ \\
\hline Known chronic lung disease, no. (\%) & 761 & $95(12.5 \%)$ & 633 & $44(7 \%)$ \\
\hline Oxygen saturation, $\%$ & 684 & $92[88-95]$ & 571 & $92[88-96]$ \\
\hline White blood cell count $/ \mathrm{mm}^{3}$ & 761 & $6655[5000-9522.5]$ & 628 & $7025[5087.5-9577.5]$ \\
\hline Creatinine, $\mathrm{mg} / \mathrm{dL}$ & 761 & $1.01[0.83-1.29]$ & 625 & $1.01[0.84-1.28]$ \\
\hline $\mathrm{LDH}, \mathrm{U} / \mathrm{L}$ & 717 & $368[275-491]$ & 240 & 320 [230.25-464.25] \\
\hline $\mathrm{CRP}, \mathrm{mg} / \mathrm{dL}$ & 761 & $11.3[5.62-19.21]$ & 615 & $9.7[3.57-17.23]$ \\
\hline Troponin I, ng/L & 144 & $13.65[6.07-43.4]$ & 51 & $18[10.5-80.5]$ \\
\hline IL-6, pg/mL & 165 & $38.1[17.6-104]$ & 96 & $100.1[32.79-495.05]$ \\
\hline D-dimer, $\mathrm{mcg} / \mathrm{mL}$ & 232 & $1.54[0.59-4.29]$ & 223 & $1.9[0.72-4]$ \\
\hline Main pulmonary artery diameter, mm & 761 & 27 [25-29] & 633 & $27[25-30]$ \\
\hline Main pulmonary diameter, no. (\%) & 761 & & 633 & \\
\hline Normal & & $541(71.1 \%)$ & & $441(69.7 \%)$ \\
\hline Mildly enlarged & & $86(11.3 \%)$ & & $58(9.2 \%)$ \\
\hline Moderately enlarged & & $98(12.9 \%)$ & & $101(15.9 \%)$ \\
\hline Severely enlarged & & $36(4.7 \%)$ & & $33(5.2 \%)$ \\
\hline Left pulmonary artery diameter, $\mathrm{mm}$ & 761 & 20 [18-22] & 633 & $21[19-23]$ \\
\hline Right pulmonary artery diameter, $\mathrm{mm}$ & 761 & $21[19-23]$ & 633 & $21[19-23]$ \\
\hline Well-aerated lung volume, $\mathrm{cm}^{3}$ & 761 & $2302[1332-3419]$ & 633 & 2283 [1383-3374] \\
\hline \multirow[t]{5}{*}{ GGO/consolidation ratio, no. (\%) } & 761 & & 633 & \\
\hline & & $9(1.2 \%)$ & & $7(1.1 \%)$ \\
\hline & & $432(56.8 \%)$ & & $328(51.8 \%)$ \\
\hline & & $145(19.1 \%)$ & & $142(22.4 \%)$ \\
\hline & & $175(23 \%)$ & & $156(24.6 \%)$ \\
\hline \multirow[t]{6}{*}{ Pneumonia score, no. (\%) } & 761 & & 633 & \\
\hline & & $9(1.2 \%)$ & & $7(1.1 \%)$ \\
\hline & & $250(32.9 \%)$ & & $181(28.6 \%)$ \\
\hline & & $363(47.7 \%)$ & & $249(39.3 \%)$ \\
\hline & & $130(17.1 \%)$ & & $151(23.9 \%)$ \\
\hline & & $9(1.2 \%)$ & & $45(7.1 \%)$ \\
\hline Outcome & & & & \\
\hline Non-survivors, no. (\%) & 761 & $182(23.9 \%)$ & 633 & $140(22.1 \%)$ \\
\hline
\end{tabular}

Data are reported as median [interquartile range, IQR], except otherwise specified

$L D H$, lactate dehydrogenase; $C R P$, C-reactive protein; $I L-6$, interleukin 6; $G G O$, ground-glass opacity

(IntelliSpace Portal 8.0, Philips Medical Systems) based on a previously defined HU threshold value [17]. Pneumonia extension was scored in 0\% (absent, score 0), 1-25\% (minimal, score 1); 26-50\% (mild, score 2); 51-75\% (moderate, score 3 ); and $>75 \%$ (severe, score 4). Qualitative features of pneumonia were scored as follows: score 0 for absent pneumonia, score 1 for prevalent ground-glass opacities (GGOs), score 2 for prevalent consolidation, and score 3 for GGOs and consolidation equally represented. The metrics of pulmonary arteries included the measurement of the MPAD at the level of its bifurcation, of the left (LPAD) and of the right pulmonary artery diameter (RPAD) at their origin. MPAD was classified according to the severity classification system for the diagnosis and prognosis of $\mathrm{PH}[13]$ in four classes: normal $(\leq 27 \mathrm{~mm}$ for females and $\leq 29 \mathrm{~mm}$ for males, according to the Framingham sex-specific normative values [16]); mild 


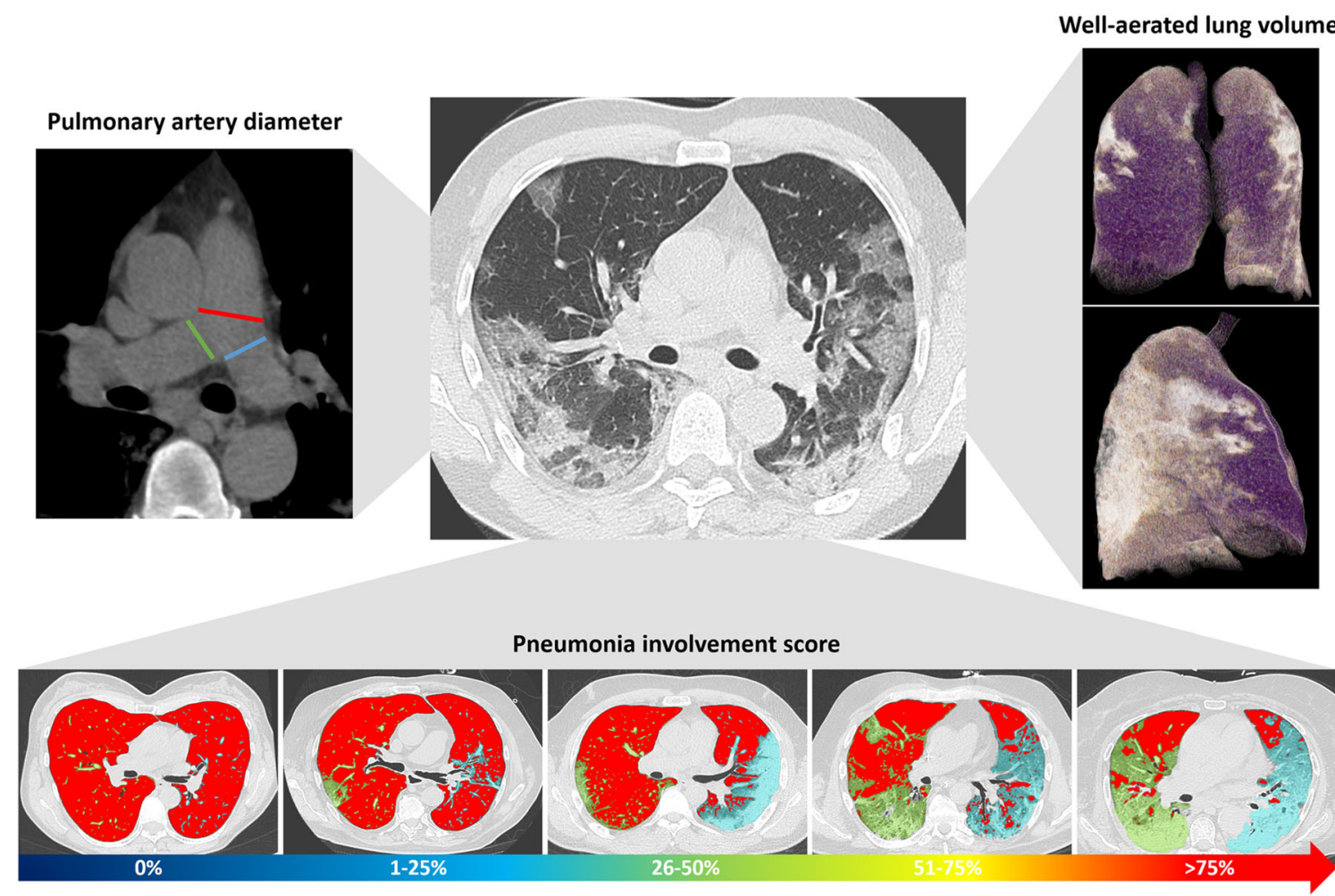

Fig. 2 Imaging parameters extracted from chest CT in COVID-19 patients. For each patient, the diameter of the main pulmonary artery (red line) was measured at the level of its bifurcation and the diameter of right and left pulmonary arteries at the level of their origin (green and blue lines, respectively) on the mediastinal window reconstruction (top left of the panel). Using dedicated software, well-aerated lung volume was

enlargement (from $>27$ to $<31 \mathrm{~mm}$ for females and $>29$ to $<31 \mathrm{~mm}$ for males); moderate enlargement (from $\geq 31$ to $34 \mathrm{~mm}$ for both sexes), and severe enlargement (>34 mm for both sexes). To assess inter-observer reliability, a second reader with 5 years of experience in cardiothoracic imaging, blinded to the measurements of the first reader, assessed MPAD and pneumonia score.

\section{Statistical analysis}

Comparisons between numerical variables were performed with the Mann-Whitney test, and comparisons between categorical variables with Fisher's exact test. $P$ values were adjusted with Bonferroni's correction to account for multiple testing. Follow-up data were censored at 40 days from hospital admission. OS was estimated with the Kaplan-Meier estimator and groups were compared with the log-rank test. The cumulative incidence of orotracheal intubation was estimated using the competing risk approach (with death without intubation as a competing event) and groups were compared with Gray's test.

Cox's regression analysis was employed for evaluating the role of MPAD classes $[13,16]$ in predicting the OS, unadjusting and adjusting for demographics and comorbidities automatically extracted (violet parenchyma in 3D lung volume rendering on the top right of the panel). Pneumonia involvement was scored from 0 to 4 (score 0 : $0 \%$; score 1: $1-25 \%$; score 2: $26-50 \%$; score 3 : $51-75 \%$ score 4: >75\%); well-aerated lung parenchyma is displayed in red, and pneumonia in green for the right lung and in blue for the left lung (bottom of the panel)

(age, sex, hypertension, diabetes, history of cardiovascular disease, and chronic lung disease). Multivariable Cox's regression analysis was employed for evaluating the simultaneous role of CT parameters (MPAD, pneumonia extension, well-aerated lung volume), laboratory tests (WBC, CRP, creatinine), demographics, and comorbidities in predicting OS. The final model was obtained with a backward variable selection with a removal significant level of 0.05 . Model calibration was performed as detailed in Supplementary Materials (ESM 1). Model discrimination was evaluated with the optimism corrected c-index. To assess the role of gender in categorized MPAD with respect to the outcome, the final Cox model was estimated adding an interaction term between the two variables.

The adjusted Cox regression analysis, the calibration curve, and the c-index of the final multivariable Cox's model were computed on the validation cohort using CT data from two independent readers.

Inter-observer reliability was assessed with Rothery's nonparametric intra-class correlation coefficient (ICC) [18] for MPAD and Cohen's kappa coefficient for the pneumonia score.

Missing data were not imputed; thus, each analysis considered only complete cases for the variables used in the analysis. 
All tests were 2-sided and the significance level was set at 0.05. Confidence intervals (CIs) were computed at a $95 \%$ level. All statistical analyses were performed using R 3.5.0 (http://www.R-project.org/). All details are reported in Supplementary Materials (ESM 1).

\section{Results}

\section{Derivation cohort: clinical and lung CT characteristics}

The median age was 69 (interquartile range IQR, 58-77) years and $537(70.6 \%)$ patients were male (Table 1). Hypertension was the most frequent comorbidity (484 patients, 63.6\%) and median oxygen saturation was $92 \%$ (IQR, $88-95 \%$ ).

At chest CT, 752 patients (98.8\%) had pneumonia, with prevalent GGOs (432 patients, 56.8\%). Pneumonia extension greater than $50 \%$ was recorded in 139 cases $(18.3 \%)$.

Median MPAD was 27 [25-29] mm. Twenty-one (2.8\%) patients were not hospitalized after emergency department evaluation, while 459 (60.3\%) patients were discharged within 40 days from admission with a median hospitalization length of 11 (IQR, 7-17) days. The cumulative incidence of orotracheal intubation at 40 days was $21.28 \%$. At 40 days, non-survivors were $182 / 761$ patients (23.9\%), with death occurring at a median time from an admission of 7 (IQR, 4-12) days. The characteristics of survivors and non-survivors are reported in Table 2. OS at 40 days was $76.08 \%$ (95\% CI: $73.11-79.17 \%)$.

\section{Derivation cohort: pulmonary enlargement and mortality}

Out of 761 patients, $86(11.3 \%)$ had mild enlargement, 98 (12.9\%) moderate enlargement, and $36(4.7 \%)$ had severe MPAD enlargement.

The cumulative incidence of orotracheal intubation was not significantly different among the classes of MPAD, despite a numerically higher incidence in patients with a severe enlargement (at 40 days [95\% CI]: for normal 21.44\% [18.09$24.99 \%$ ], for mild enlargement $17.44 \%$ [10.27-26.19\%], for moderate enlargement $21.43 \%$ [13.91-30.02\%], and for severe enlargement $27.78 \%$ [14.34-42.96\%]; Gray's test $p=0.609$ ).

The mortality rate was $25.6 \%$ (22/86), $38.8 \%$ (38/98), and $47.2 \%$ (17/36) in patients with mild, moderate, and severe MPAD enlargement, respectively, while it was $19.4 \%$ (105/ 541 ) in patients with normal MPAD (global Fisher's exact test $p<0.001)$. Indeed, the classes of MPAD were associated with a different risk of death (40-day overall survival [95\% CI]: for normal $80.56 \%$ [77.32-83.99\%], for mild enlargement $74.40 \%[65.72-84.23 \%]$, for moderate enlargement $61.22 \%$ [52.30-71.67\%], and for severe enlargement $52.78 \%$
[38.75-71.89\%]; log-rank test $p<0.001$ ) (Fig. 3a). At Cox's regression analysis, moderate to severe MPAD enlargement $(\geq 31 \mathrm{~mm})$ resulted to predict a significantly reduced overall survival with respect to normal MPAD (hazard ratio, HR [95\% CI]: mild enlargement vs normal 1.387 [0.876-2.196], $p=0.163$; moderate enlargement vs normal 2.311 [1.594$3.350], p<0.001$; severe enlargement vs normal 3.001 [1.797-5.010], $p<0.001)$. Similar results were obtained also at unadjusted Cox's regression analysis (Table S1). Therefore, in the subsequent analyses, the MPAD was dichotomized into moderate-severe MPAD enlargement $(\geq 31 \mathrm{~mm})$ vs normalmild MPAD enlargement $(<31 \mathrm{~mm})$. The percentage of moderate-severe MPAD resulted to be similar between genders (M: $18.6 \%$ vs F: $15.2 \%, p=1$ ) (Table S2). The new categorization achieved HR $=1.741$ (95\% CI: $1.253-2.418$, $p<0.001$ ) at adjusted Cox's regression (at unadjusted analysis: $\mathrm{HR}=2.367$ [1.725; 3.249], $p<0.001$ ) (Fig. 3b).

Moderate-severe MPAD enlargement remained associated with a higher risk of death at multiple Cox's regression analysis also when pneumonia extension, wellaerated lung volume, laboratory tests, demographics, and comorbidities were considered (HR [95\% CI]: 1.592 [1.154-2.196], $p=0.005$ ) (Table 3). Figure 4 reports two exemplifying cases with normally and moderately enlarged MPAD, respectively. Besides MPAD, the other variables associated with higher risk of mortality in the model were old age (HR [95\% CI]: 1.063 [1.052-1.075], $p<0.001$ ), male gender (1.586 [1.079-2.329], $p=0.019$ ), higher creatinine (2.468 [1.824-3.339], $p<0.001)$, and greater pneumonia score (1.397 [1.084-1.799], $p=$ 0.010 ), while greater well-aerated lung volume was a protective factor $(0.699$ [0.581-0.841], $p<0.001)$. The final model showed a good calibration (Figure S1) and discrimination (c-index [95\% CI]: 0.826 [95\% CI: 0.796-0.851]).

An interaction term between MPAD and gender was included in the final multiple Cox regression model and resulted not to be significant $(p=0.724)$, thus supporting that MPAD has a similar role in both sexes.

\section{Validation of enlarged MPAD as a predictor of mortality}

In the validation cohort, the median age was 69 years (IQR, 59-77 years) and $421(66.5 \%)$ patients were males (Table 1). Hypertension was the most frequent comorbidity (285 patients, $45 \%$ ) and median oxygen saturation was $92 \%$ (IQR, $88-96 \%)$.

Most of the patients $(626,98.9 \%)$ had pneumonia at chest CT, with prevalent GGOs (328 patients, 51.8\%). Thirty-one percent of cases (196) had lung involvement greater than 50\%.

Mild, moderate, and severe MPAD enlargement was found in $58(9.2 \%), 101(15.9 \%)$, and $33(5.2 \%)$ patients, respectively. 
Table 2 Clinical and CT features of survivors vs non-survivors in the derivation cohort

\begin{tabular}{|c|c|c|c|c|c|}
\hline \multirow[b]{2}{*}{ Variables } & \multicolumn{2}{|c|}{ Survivors (40-days FU) } & \multicolumn{2}{|c|}{ Non-survivors (40-days FU) } & \multirow[b]{2}{*}{ adj. $p$ value } \\
\hline & $N$ & Median [IQR] & $N$ & Median [IQR] & \\
\hline Age, years & 579 & $64.9[55.66-73.96]$ & 182 & 76.97 [71.37-82.49] & $<0.001$ \\
\hline Male & 579 & $396(68.4 \%)$ & 182 & $141(77.5 \%)$ & 0.396 \\
\hline Hypertension, no. (\%) & 579 & $340(58.7 \%)$ & 182 & $144(79.1 \%)$ & $<0.001$ \\
\hline Diabetes, no. (\%) & 579 & $99(17.1 \%)$ & 182 & $51(28 \%)$ & 0.037 \\
\hline Known cardiovascular disease, no. $(\%)$ & 579 & $58(10 \%)$ & 182 & $37(20.3 \%)$ & 0.009 \\
\hline Known chronic lung disease, no. (\%) & 579 & $60(10.4 \%)$ & 182 & $35(19.2 \%)$ & 0.057 \\
\hline Oxygen saturation, $\%$ & 579 & $93[90-96]$ & 182 & 88 [83-92] & $<0.001$ \\
\hline White blood cell count $/ \mathrm{mm}^{3}$ & 579 & $6400[4900-9100]$ & 182 & $7250[5340-11,020]$ & 0.051 \\
\hline Creatinine, mg/dL & 579 & $0.97[0.8-1.19]$ & 182 & $1.26[1-1.78]$ & $<0.001$ \\
\hline $\mathrm{LDH}, \mathrm{U} / \mathrm{L}$ & 553 & 347 [258-449] & 164 & $462.5[354.5-618.5]$ & $<0.001$ \\
\hline $\mathrm{CRP}, \mathrm{mg} / \mathrm{dL}$ & 579 & $10.4[5.07-18.18]$ & 182 & $13.96[8.43-21.12]$ & 0.003 \\
\hline Troponin I, ng/L & 125 & $12.1[5.4-33.5]$ & 19 & $76.2[49.7-514.1]$ & $<0.001$ \\
\hline IL-6, pg/mL & 150 & 33.8 [14.77-92.02] & 15 & $124[69.2-240]$ & 0.011 \\
\hline D-dimer, mcg/mL & 203 & $1.32[0.59-3.88]$ & 29 & $3.92[0.88-9.22]$ & 0.445 \\
\hline Main pulmonary artery diameter, $\mathrm{mm}$ & 579 & 27 [24-29] & 182 & $28[26-31]$ & $<0.001$ \\
\hline Left pulmonary artery diameter, $\mathrm{mm}$ & 579 & 20 [18-22] & 182 & $21.3[20-24]$ & $<0.001$ \\
\hline Right pulmonary artery diameter, mm & 579 & 20 [18-23] & 182 & $22[20-25]$ & $<0.001$ \\
\hline Well-aerated lung volume, $\mathrm{cm}^{3}$ & 579 & 2506 [1564-3609] & 182 & 1520.5 [1009-2544] & $<0.001$ \\
\hline \multirow[t]{5}{*}{ GGO/consolidation ratio, no. (\%) } & 579 & & 182 & & 1.000 \\
\hline & & $9(1.6 \%)$ & & $0(0 \%)$ & \\
\hline & & $318(54.9 \%)$ & & $114(62.6 \%)$ & \\
\hline & & $117(20.2 \%)$ & & $28(15.4 \%)$ & \\
\hline & & $135(23.3 \%)$ & & $40(22 \%)$ & \\
\hline \multirow[t]{6}{*}{ Pneumonia score, no. (\%) } & 579 & & 182 & & $<0.001$ \\
\hline & & $9(1.6 \%)$ & & $0(0 \%)$ & \\
\hline & & $220(38 \%)$ & & $30(16.5 \%)$ & \\
\hline & & $268(46.3 \%)$ & & $95(52.2 \%)$ & \\
\hline & & $77(13.3 \%)$ & & $53(29.1 \%)$ & \\
\hline & & $5(0.9 \%)$ & & $4(2.2 \%)$ & \\
\hline
\end{tabular}

Data are reported as median [IQR], except otherwise specified

$I Q R$, interquartile range; adj. $p$ value, adjusted $p$ value with Bonferroni's correction; $L D H$, lactate dehydrogenase; $C R P$, C-reactive protein; $I L-6$, interleukin 6; $G G O$, ground-glass opacity

Inter-reader agreement was excellent for both MPAD (ICC [95\% CI]: 0.937 [0.931-1]) and pneumonia score (Cohen's kappa [95\% CI]: 0.886 [0.856-0.916]).

The mortality rate of the validation cohort was $22.1 \%$ (140/ 633 patients). OS at 40 days was $77.69 \%$ (95\% CI $=74.50$ $81.03 \%)$.

At Cox's regression analysis adjusted with respect to demographics and comorbidities, the risk of mortality associated with moderate-severe MPAD enlargement was slightly higher than in the derivation cohort (HR [95\% CI]: 2.524 [1.784$3.571], p<0.001)$. The discrimination of the model obtained in the derivation cohort was confirmed, using CT measurement from both the first and second reader (c-index [95\% CI]: 0.789 [0.758-0.823] and 0.790 [0.753-0.825], respectively).

\section{Discussion}

The main finding of our study is that moderate-severe enlargement of the pulmonary artery diameter (MPAD $\geq 31 \mathrm{~mm}$ ) at chest CT performed within $72 \mathrm{~h}$ from the admission is an independent predictor of death in COVID-19 patients. The multivariable model, coming from the analysis of 761 consecutive COVID-19 patients admitted to three third-level hospitals in Northern Italy, was confirmed on an external validation cohort including 633 patients from four other third-level hospitals in the same Italian territory.

Enlargement of MPAD was associated with a higher rate of in-hospital mortality: $19.4 \%(105 / 541)$ in patients with normal MPAD and 25.6\% (22/86), 38.8\% (38/98), and 47.2\% (17/36) 


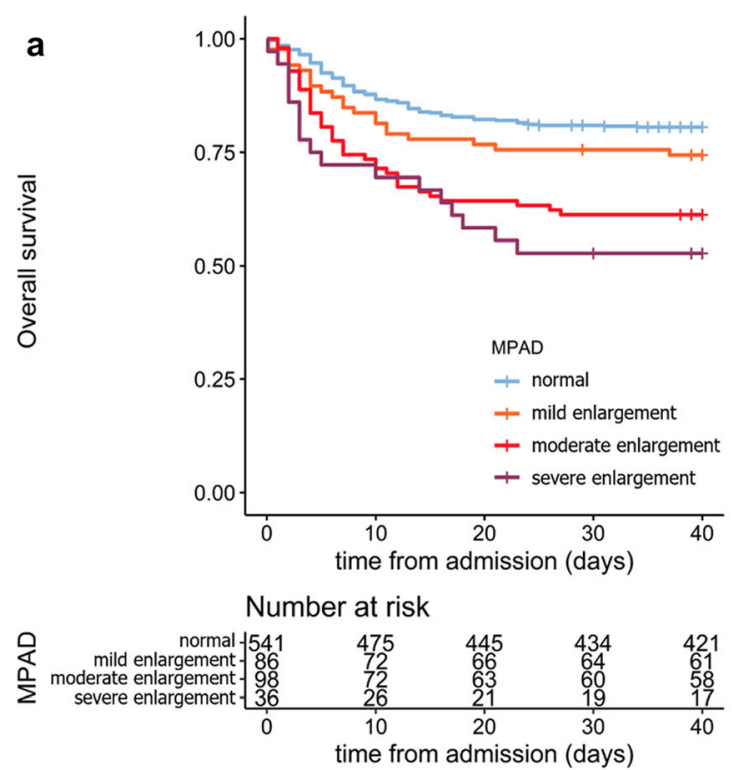

Fig. 3 Overall survival in COVID-19 patients according to four-tier (a) and two-tier (b) classification of main pulmonary artery enlargement. The Kaplan-Meier curves show the overall survival in COVID-19 patients according to the four-tier classification system of MPAD classification (a) [13]. The four classes of MPAD were associated with a different risk of death (40-day overall survival [95\% CI]: $80.56 \%$ [77.32-83.99\%] for normal MPAD, $74.40 \%$ [65.72-84.23\%] for mild enlargement, $61.22 \%$ [52.30-71.67\%] for moderate enlargement, $52.78 \%$ [38.75-71.89\%] for severe enlargement; log-rank test $p<0.001)$. In $\mathbf{b}$ are reported the KaplanMeier curves (solid lines) and the adjusted Kaplan-Meier curves (dashed

in patients with mild, moderate, and severe MPAD enlargement (global Fisher's exact test $p<0.001$ ).

Enlarged pulmonary artery on chest CT scan was previously demonstrated to be associated with pulmonary hypertension in other clinical settings before COVID-19 outbreak $[12,13,19]$; it is considered a consequence of elevated pressure, reflecting disease severity [13] and duration [20].

MPAD $\geq 31 \mathrm{~mm}$ was previously found to have excellent specificity (from 80 to $98 \%$ ) and positive predictive value (from 85 to 98\%) for the diagnosis of $\mathrm{PH}$ [13], with a 2-3fold increased risk of mortality than in patients with normal MPAD [13].

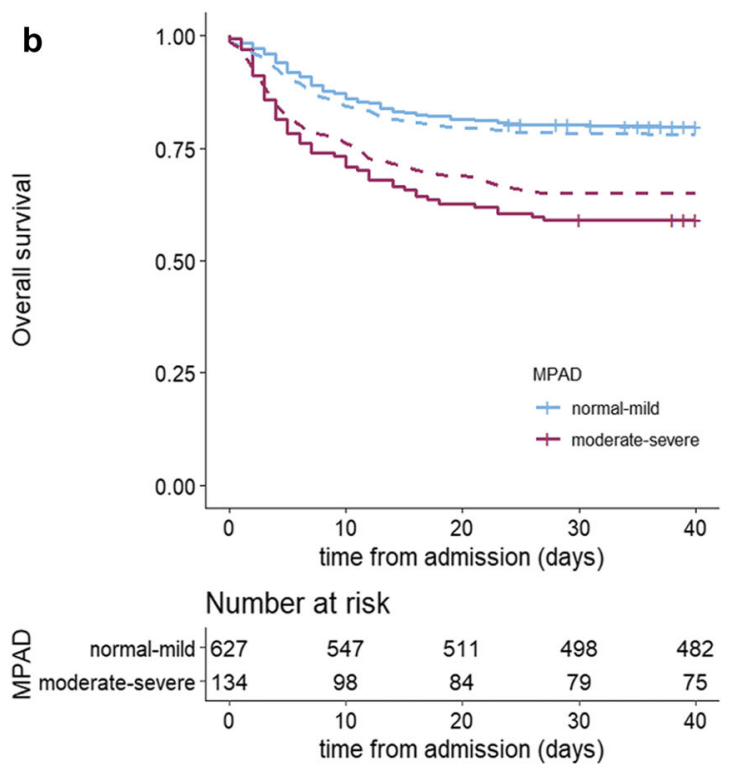

lines) with inverse probability weighting based on propensity score (detailed in Supplementary Materials ESM 1) showing the overall survival in COVID-19 patients according to two-tier MPAD enlargement (moderate-severe vs normal-mild). The two classes of MPAD enlargement were associated with a different risk of death (40-day OS [95\% CI]: $79.74 \%$ [76.65-82.95\%] for normal-mild enlargement MPAD and $58.96 \%$ [51.19-67.90\%] for moderate-severe enlargement; log-rank test $p<0.001$; adjusted log-rank test with inverse probability weighting based on propensity score $p=0.002$ )

Our results, based on a single time-point CT examination, do not allow to assert if the enlargement of MPAD is acute or not. However, the statistical adjustment for demographics and comorbidities suggested that the observed phenomenon is more likely an acute complication of COVID-19 pneumonia. Moreover, a previous CT study [21] on a limited sample size (44 COVID-19 patients) showed an MPAD enlargement with respect to a previous $\mathrm{CT}$ examination in non-survivors, further supporting the hypothesis that MPAD enlargement occurred in response to increased peripheral resistance for COVID-19associated lung endothelial injury $[3,22,23]$.
Table 3 Cox's multivariable regression model for predicting overall survival estimated in the derivation cohort (c-index [95\% $\mathrm{CI}]=0.826[0.796-0.851]$; baseline survival at 28 days $=$ $99.92 \%$ )

\begin{tabular}{lcrr}
\hline & \multicolumn{3}{l}{ Derivation cohort $(n=761)$} \\
\cline { 2 - 4 } Variable & Coefficient & HR [95\% CI] & $p$ value \\
\hline Age, years & 0.061 & $1.063[1.052-1.075]$ & $<0.001$ \\
Male & 0.461 & $1.586[1.079-2.329]$ & 0.019 \\
Creatinine, mg/dL & 0.903 & $2.468[1.824-3.339]$ & $<0.001$ \\
Pneumonia score, $\%$ & 0.334 & $1.397[1.084-1.799]$ & 0.010 \\
Well-aerated lung volume, $\mathrm{dm}^{3}$ & -0.358 & $0.699[0.581-0.841]$ & $<0.001$ \\
Moderate-severe main pulmonary artery diameter & 0.465 & $1.592[1.154-2.196]$ & 0.005 \\
$\quad$ enlargement $(\geq 31 \mathrm{~mm})$ & & & \\
\hline
\end{tabular}




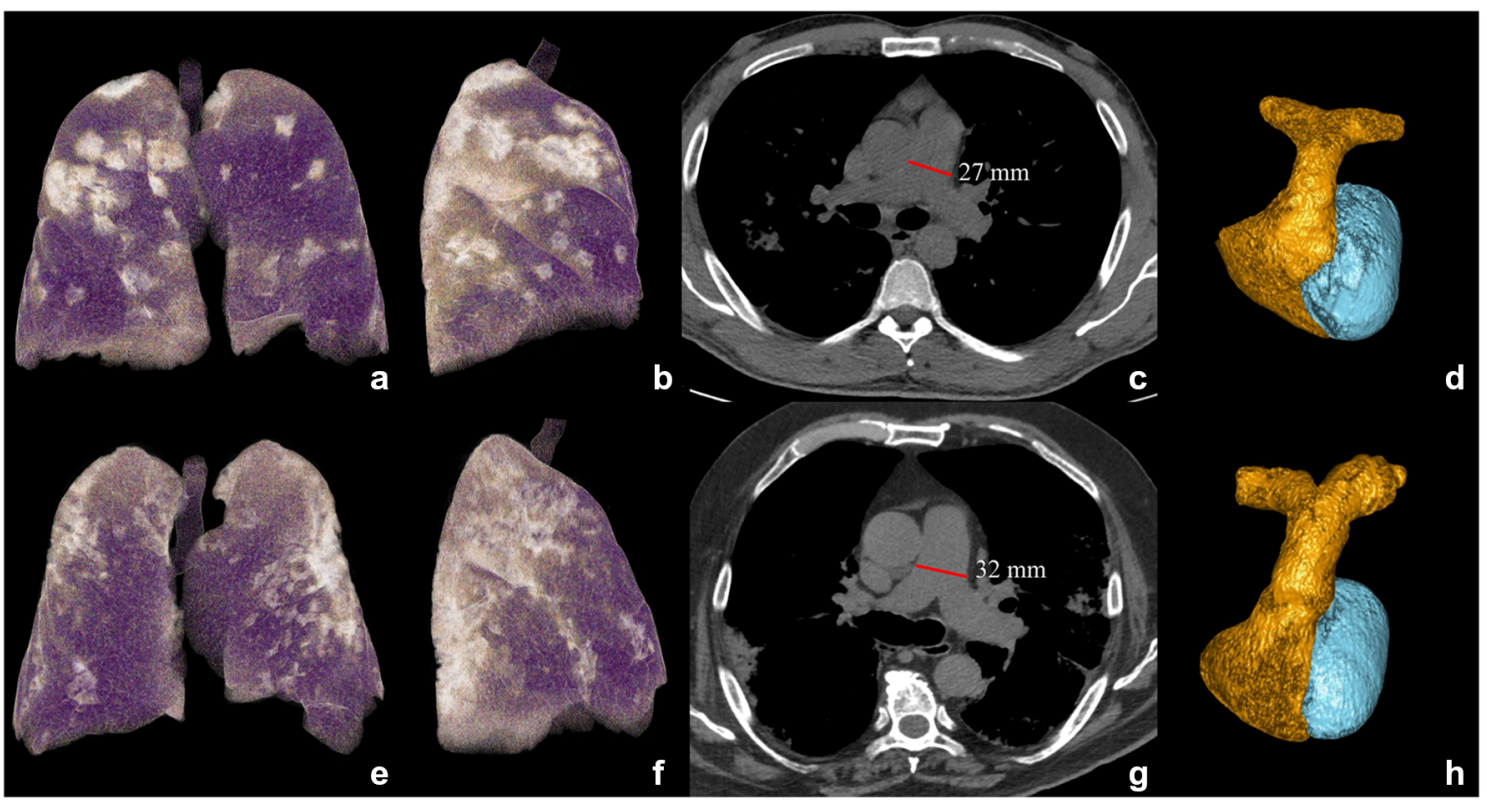

Fig. 4 Exemplifying cases: a survivor (top) and non-survivor (bottom) patients. CT 3D volume rendering of lungs in antero-posterior view (a, e) and lateral view $(\mathbf{b}, \mathbf{f}), \mathrm{CT}$ axial images with mediastinal window $(\mathbf{c}, \mathbf{g})$ for main pulmonary artery diameter (MPAD) measurement (red lines in $\mathbf{c}, \mathbf{g}$ ), and $3 \mathrm{D}$ volumetric reconstruction of pulmonary arteries $(\mathbf{d}, \mathbf{h})$ in a survivor (top) and a non-survivor (bottom) COVID-19 patient, both males of 74 and 76 years, respectively, both with history of hypertension and treated ischemic cardiomyopathy, both suffering from fever $\left(>37.5^{\circ} \mathrm{C}\right)$ and caught from 7 days. At the admission, $\mathrm{WBC} / \mathrm{mm}^{3}$ were 4.36 and 3.8,

In the setting of non-COVID-19-related acute respiratory distress syndrome [24], $\mathrm{PH}$ resulted in highly prevalent (almost 50\%) and entails higher risk of cardiac failure, shock [25, $26]$, and mortality [26, 27]. The etiology of acute PH is considered to be multifactorial, being related to the refractory hypoxemia, vasoconstriction, pulmonary edema, and microvascular thrombosis [28].

In the COVID-19 setting, where angiotensin-converting enzyme 2 on epithelial cells was recognized as a functional receptor for SARS-CoV-2, low levels of angiotensin and decreased downregulation of angiotensin II may contribute to determine acute $\mathrm{PH}$ by increasing pulmonary vasoconstriction [29]. Moreover, pulmonary small-vessel thrombosis could be the main cause of PH in COVID-19 pneumonia. In the autopsy series of COVID-19 patients [1, 2, 30], hallmarks of classic ARDS were not prominent [30], while a pauci-inflammatory thrombogenic vasculopathy [30], with small-vessel thrombosis $[1,2,23]$, was reported as a common finding. In our cohort, similar to previous studies $[4,31]$, higher levels of IL-6, CRP, $\mathrm{LDH}$, D-dimer, and troponin were found in non-survivors compared to survivors, further supporting the hypothesis of microvascular COVID-19 lung thrombo-inflammatory syndrome [4].

The measurement of the MPAD on chest CT is easy, fast (few seconds), highly reproducible, does not require any
CRP 0.93 and $1.1 \mathrm{mg} / \mathrm{dL}, \mathrm{LDH} 295$ and $378 \mathrm{U} / \mathrm{L}$, and creatinine 0.93 and $1.1 \mathrm{mg} / \mathrm{dL}$ in survivor and non-survivor, respectively. The oxygen saturation was $90 \%$ and $94 \%$ for survivor and non-survivor, respectively, with higher well-aerated lung volume in the survivor patient $(3882 \mathrm{~mL}$ vs $2382 \mathrm{~mL}$, violet parenchyma on $3 \mathrm{D}$ lung volume rendering in $\mathbf{a}, \mathbf{b}, \mathbf{e}, \mathbf{f})$ with pneumonia (bright parenchyma in $\mathbf{a}, \mathbf{b}, \mathbf{e}, \mathbf{f}$ ), involving from 25 to $50 \%$ of lung volume in both cases. Pulmonary artery diameter was normal $(27 \mathrm{~mm})$ in the survivor $(\mathbf{c}, \mathbf{d})$ while it was enlarged $(32 \mathrm{~mm})$ in the nonsurvivor $(\mathbf{g}, \mathbf{h})$ who died 9 days after hospital admission

dedicated software, and could be a useful quantitative marker to be integrated into models for risk stratification. Moreover, the detection of enlarged MPAD on chest $\mathrm{CT}$, being a known sign of $\mathrm{PH}$, may have a potential impact on patient management and treatment. In fact, patients with $\mathrm{PH}$ may rapidly deteriorate with RV dysfunction and worsening oxygenation for ventilation-perfusion mismatch. In patients with severe pneumonia and dilated MPAD, alveolar ventilation could be optimized to limit hypoxic vasoconstriction, and prone ventilation preferred for minimizing positive end-expiratory pressure and hemodynamic impact [7]. Close monitoring of pulmonary vascular resistance should be considered to optimize inotropic support and pulmonary vasodilatation [7, 32], decreasing RV afterload.

The limitation of the present study was the lack of data about pulmonary artery pressure estimated by right heart catheterization or echocardiography. Despite echocardiography being commonly used to investigate pulmonary hypertension, it was not routinely performed in all COVID-19 patients. Moreover, its diagnostic accuracy is affected by body habitus, heart rate, acoustic window, operator's experience [33], and notably by the presence of significant lung disease [5].

In our study, no differences in orotracheal intubation rate were found between patients with normal or enlarged MPAD, 
despite numerically higher rate in severely enlarged MPAD. This result might be influenced by bias of treatment in overwhelmed hospitals closely related to the scarcity of resources.

Non-survivor rates in our derivation and validation cohorts (23.9\% and $22.1 \%$, respectively) are consistent with the mortality rate recorded in Italy during the COVID-19 outbreak [34], suggesting that our cohort could be considered a representative sample of the Italian COVID-19 population.

In conclusion, in the present study, on a large cohort of COVID-19 patients from seven third-level hospitals from Northern Italy, we found that enlarged MPAD measured on chest $\mathrm{CT}$ at admission is an independent predictor of mortality. The MPAD can be easily measured on chest CT performed for pneumonia extension evaluation, offering additional prognostic information, and helping clinicians in patients' management.

Supplementary Information The online version contains supplementary material available at https://doi.org/10.1007/s00330-020-07622-x.

Funding The authors state that this work has not received any funding.

\section{Compliance with ethical standards}

Guarantor The scientific guarantor of this publication is prof. Antonio Esposito.

Conflict of interest The authors of this manuscript declare no relationships with any companies whose products or services may be related to the subject matter of the article.

Statistics and biometry Prof. Paola MV Rancoita kindly provided statistical advice for this manuscript.

Informed consent Written informed consent was obtained from all subjects (patients) in this study.

Ethical approval Institutional Review Board approval was obtained.

\section{Methodology \\ - retrospective \\ - observational \\ - multicenter study}

Open Access This article is licensed under a Creative Commons Attribution 4.0 International License, which permits use, sharing, adaptation, distribution and reproduction in any medium or format, as long as you give appropriate credit to the original author(s) and the source, provide a link to the Creative Commons licence, and indicate if changes were made. The images or other third party material in this article are included in the article's Creative Commons licence, unless indicated otherwise in a credit line to the material. If material is not included in the article's Creative Commons licence and your intended use is not permitted by statutory regulation or exceeds the permitted use, you will need to obtain permission directly from the copyright holder. To view a copy of this licence, visit http://creativecommons.org/licenses/by/4.0/.

\section{References}

1. Buja LM, Wolf DA, Zhao B et al (2020) The emerging spectrum of cardiopulmonary pathology of the coronavirus disease 2019 (COVID-19): report of 3 autopsies from Houston, Texas, and review of autopsy findings from other United States cities. Cardiovasc Pathol 48:107233

2. Fox SE, Akmatbekov A, Harbert JL, Li G, Brown JQ, Vander Heide RS (2020) Pulmonary and cardiac pathology in African American patients with COVID-19: an autopsy series from New Orleans. Lancet Respir Med 8(7):681-686

3. Varga Z, Flammer AJ, Steiger P et al (2020) Endothelial cell infection and endotheliitis in COVID-19. Lancet 395:1417-1418

4. Ciceri F, Beretta L, Scandroglio AM et al (2020) Microvascular COVID-19 lung vessels obstructive thromboinflammatory syndrome (MicroCLOTS): an atypical acute respiratory distress syndrome working hypothesis. Crit Care Resusc 22(2):95-97

5. Li Y, Li H, Zhu S et al (2020) Prognostic value of right ventricular longitudinal strain in patients with COVID-19. JACC Cardiovasc Imaging. https://doi.org/10.1016/j.jcmg.2020.04.014

6. D'Andrea A, Scarafile R, Riegler L et al (2020) Right ventricular function and pulmonary pressures as independent predictors of survival in patients with COVID-10 pneumonia. JACC Cardiovasc Imaging. https://doi.org/10.1016/j.jcmg.2020.06.004

7. Milligan GP, Alam A, Guerrero-Miranda C (2020) Recognizing right ventricular dysfunction in coronavirus disease-2019-related respiratory illness. J Card Fail. https://doi.org/10.1016/j.cardfail. 2020.05.003

8. Bikdeli B, Madhavan MV, Jimenez D et al (2020) COVID-19 and thrombotic or thromboembolic disease: implications for prevention, antithrombotic therapy, and follow-up: JACC state-of-the-art review. J Am Coll Cardiol 75:2950-2973

9. Eberhard M, Milanese G, Ho M et al (2019) Pre-procedural CT angiography inferior vena cava measurements: a predictor of mortality in patients undergoing transcatheter aortic valve implantation. Eur Radiol 29:975-984

10. Ai T, Yang Z, Hou H et al (2020) Correlation of chest CT and RTPCR testing in coronavirus disease 2019 (COVID-19) in China: a report of 1014 cases. Radiology. https://doi.org/10.1148/radiol. 2020200642:200642

11. Liu F, Zhang Q, Huang C et al (2020) CT quantification of pneumonia lesions in early days predicts progression to severe illness in a cohort of COVID-19 patients. Theranostics 10:5613-5622

12. Corson N, Armato SG 3rd, Labby ZE, Straus C, Starkey A, Gomberg-Maitland M (2014) CT-based pulmonary artery measurements for the assessment of pulmonary hypertension. Acad Radiol 21:523-530

13. Truong QA, Bhatia HS, Szymonifka J et al (2018) A four-tier classification system of pulmonary artery metrics on computed tomography for the diagnosis and prognosis of pulmonary hypertension. $\mathrm{J}$ Cardiovasc Comput Tomogr 12:60-66

14. Tan RT, Kuzo R, Goodman LR, Siegel R, Haasler GB, Presberg KW (1998) Utility of CT scan evaluation for predicting pulmonary hypertension in patients with parenchymal lung disease. Medical College of Wisconsin Lung Transplant Group. Chest 113:1250 1256

15. Melzig C, Worz S, Egenlauf B et al (2019) Combined automated $3 \mathrm{D}$ volumetry by pulmonary CT angiography and echocardiography for detection of pulmonary hypertension. Eur Radiol 29:60596068

16. Truong QA, Massaro JM, Rogers IS et al (2012) Reference values for normal pulmonary artery dimensions by noncontrast cardiac computed tomography: the Framingham Heart Study. Circ Cardiovasc Imaging 5:147-154 
17. Colombi D, Bodini FC, Petrini M et al (2020) Well-aerated lung on admitting chest $\mathrm{CT}$ to predict adverse outcome in COVID-19 pneumonia. Radiology. https://doi.org/10.1148/radiol.2020201433: 201433

18. Rothery P (1979) A nonparametric measure of intraclass correlation. Biometrika 66:629-639

19. Mohamed Hoesein FA, Besselink T, Pompe E et al (2016) Accuracy of CT pulmonary artery diameter for pulmonary hypertension in end-stage COPD. Lung 194:813-819

20. Boerrigter B, Mauritz GJ, Marcus JT et al (2010) Progressive dilatation of the main pulmonary artery is a characteristic of pulmonary arterial hypertension and is not related to changes in pressure. Chest 138:1395-1401

21. Spagnolo P, Cozzi A, Foa RA et al (2020) CT-derived pulmonary vascular metrics and clinical outcome in COVID-19 patients. Quant Imaging Med Surg 10:1325-1333

22. Patel BV, Arachchillage DJ, Ridge CA et al (2020) Pulmonary angiopathy in severe COVID-19: physiologic, imaging and hematologic observations. Am J Respir Crit Care Med. https://doi.org/ 10.1164/rccm.202004-1412OC

23. Grosse C, Grosse A, Salzer HJF, Dünser MW, Motz R, Langer R (2020) Analysis of cardiopulmonary findings in COVID-19 fatalities: high incidence of pulmonary artery thrombi and acute suppurative bronchopneumonia. Cardiovasc Pathol 49:107263

24. Zochios V, Parhar K, Tunnicliffe W, Roscoe A, Gao F (2017) The right ventricle in ARDS. Chest 152:181-193

25. Repesse X, Charron C, Vieillard-Baron A (2015) Acute cor pulmonale in ARDS: rationale for protecting the right ventricle. Chest 147:259-265
26. Boissier F, Katsahian S, Razazi K et al (2013) Prevalence and prognosis of cor pulmonale during protective ventilation for acute respiratory distress syndrome. Intensive Care Med 39:1725-1733

27. Osman D, Monnet X, Castelain V et al (2009) Incidence and prognostic value of right ventricular failure in acute respiratory distress syndrome. Intensive Care Med 35:69-76

28. Price LC, McAuley DF, Marino PS, Finney SJ, Griffiths MJ, Wort SJ (2012) Pathophysiology of pulmonary hypertension in acute lung injury. Am J Physiol Lung Cell Mol Physiol 302:L803-L815

29. Farha S (2020) COVID-19 and pulmonary hypertension. Cleve Clin J Med. https://doi.org/10.3949/ccjm.87a.ccc021

30. Magro C, Mulvey JJ, Berlin D et al (2020) Complement associated microvascular injury and thrombosis in the pathogenesis of severe COVID-19 infection: a report of five cases. Transl Res 220:1-13

31. Guo T, Fan Y, Chen M et al (2020) Cardiovascular implications of fatal outcomes of patients with coronavirus disease 2019 (COVID19). JAMA Cardiol. https://doi.org/10.1001/jamacardio.2020.1017

32. Jentzer JC, Mathier MA (2016) Pulmonary hypertension in the intensive care unit. J Intensive Care Med 31:369-385

33. Janda S, Shahidi N, Gin K, Swiston J (2011) Diagnostic accuracy of echocardiography for pulmonary hypertension: a systematic review and meta-analysis. Heart 97:612-622

34. Grasselli G, Zangrillo A, Zanella A et al (2020) Baseline characteristics and outcomes of 1591 patients infected with SARS-CoV-2 admitted to ICUs of the Lombardy region, Italy. JAMA. https://doi. org/10.1001/jama.2020.5394

Publisher's note Springer Nature remains neutral with regard to jurisdictional claims in published maps and institutional affiliations. 